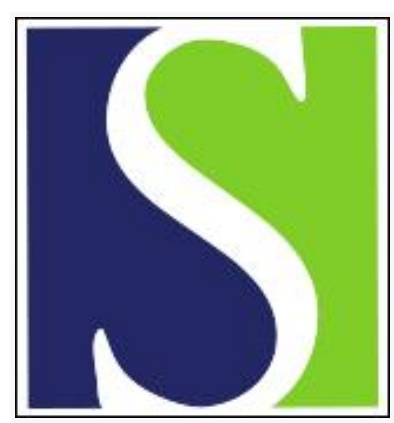

Scand J Work Environ Health 2000;26(2):85-86

https://doi.org/10.5271/sjweh.515

Issue date: Apr 2000

Electric hypersensitivity and neurophysiological effects of cellular phones - facts or needless anxiety?

by Härmä $M$

This article in PubMed: www.ncbi.nlm.nih.gov/pubmed/10817371

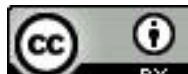




\section{Electric hypersensitivity and neurophysiological effects of cellular phones - facts or needless anxiety?}

The use of visual display screens and handheld mobile phones, or cellular phones as they are called, has increased dramatically in recent years. In Finland, $70 \%$ of the population has a mobile telephone, and almost everyone is daily exposed to the weak electromagnetic fields emitted by the video displays of televisions, personal computers, and other such devices. In addition, the users of mobile phones - and especially the users of video-screen-based devices - are younger than ever. At work, the amount of new technology has increased steeply, and mobile technology has made it possible to work without limitations.

It is understandable that the extensive use of new equipment and the exposure to electromagnetic fields have increased concerns about the possible biological or adverse effects. The radiofrequency (RF) power emitted by mobile phones is too low to cause significant warming of absorbing tissues (1). As a consequence, increasing interest has focused on the nonthermal effects of RF radiation, especially on the possible functional disturbances of the brain. Thus far, only a few studies have been published concerning the possible effects of cellular phones on the electroencephalographic (EEG) activity of humans. In this issue, Hietanen and her co-workers (2) studied the effects of RF electromagnetic fields emitted from cellular phones on the EEG activity of humans while awake. A single-blind, placebocontrolled experiment was used. To everyone's relief, the findings suggest that exposure to RF fields emitted by 5 different cellular phones, operating at $900 \mathrm{MHz}$ or $1800 \mathrm{MHz}$, had no abnormal EEG effects on humans.

"Hypersensitivity" to electricity is a separate and controversial condition. Many workers complain of its symptoms, such as redness and burning of the skin and face, pricking, pain or dryness of the throat and mouth, abdominal pain, lack of concentration, and headache, mainly from exposure to video screens. The persons suffering from the condition are very difficult to treat, and many of them are often on sick leave even though "hypersensitivity to electricity" is not an accepted diagnosis. Most earlier double-blind provocation studies have shown that such patients have not been able to determine whether or not they have been subject to a field or sham exposure. However, most of the studies have been performed in the laboratory and may not have been representative of normal living conditions. In this issue, Flodin and his co-workers (3) performed a double-blind provocation study in the homes or workplaces of patients, rather than in the laboratory, while also taking into consideration the long possible latency of "electric hypersensitivity" symptoms. However, the patients, selected for study because of distinct reactions from electric equipment, were no better than their control group in deciding whether or not they were exposed to electric and magnetic fields.

The new studies offer us some relief, but they also point out the need for new and objective information. Vast economic interests are related to the production and use of new technology. Any doubts or hints of possible health or "brain" effects need urgent research, which also should not have any conflict of interest with the manufacturers of such technology. From the point of view of technology producers, early and objective data on the safety of new products help to respond to the resistance and prejudices against the distribution of new technology, for example, the rapid installation of antennas for cellular phone base stations, which are already being opposed by new groups of activists.

Research on the biological effects of electromagnetic or electric fields also places high requirements on the quality of experimental research. The observed negative results should not raise any 
methodological speculations. On the other hand, the used methods and exposure parameters vary widely (4) and minor biological and neurophysiological effects are always difficult to detect. The lack of knowledge on biological mechanisms and the lack of information on the biologically "significant" effects of the selected output measures make adequate power estimations extremely difficult. These estimations would be badly needed to aid the selection of correct designs and sample sizes and to hinder later speculations on the quality of the mostly "negative" studies.

Biological research on electromagnetic fields keeps us alert. Recently, a well-done experimental study suggested that the RF fields emitted by GSM telephones have a facilitating effect on cognitive processing, especially in tasks that require attention or cognitive manipulation in working memory (5). At the same time, the European Union parlament arranged a discussion forum with the title "Are Wireless Waves Scrambling Our Brains?" Both examples indicate the urgent need for objective scientific data on the short- and long-term effects of electromagnetic fields on human brain function and health.

\section{References}

1. Anderson V, Joyner KH. Specific absorption rate levels measured ina phantom head exposed to radio frequency transmissions from analog hand-held mobile phones. Bioelectromagnetics $1995 ; 16: 60$-9

2. Hietanen M, Kovala T, Hämäläinen A-M. Human brain activity during exposure to radiofrequency field emitted by cellular phones. Scand J Work Environ Health 2000;2:87-92.

3. Flodin U, Seneby A. Tegenfeldt C. Provocation of electric hypersensitivity under everyday conditions. Scand J Work Environ Health 2000;2;93-8.

4. Juutilainen J, de Seze R. Biological effects of amplitude-modulated radiofrequency radiation. Scand J Work Environ Health 1998;24(4):245-54.

5. Koivisto M, Revensuo A, Krause C, Haarala C, Sillanmäki L, Laine M, Hämäläinen H. Effects of $902 \mathrm{MHz}$ electromagnetic field emitted by cellular telephones on response times in humans. NeuroReport $2000 ; 11(2): 413-5$.

\section{Mikko Härmä, Research Professor}

Editor in Chief, Scandinavian Journal of Work, Environment \& Health 